\title{
TRANSVERSE-SIGMOID SINUS DURAL ARTERIOVENOUS FISTULA PRESENTING WITH RECURRENT TRANSIENT ISCHAEMIC ATTACK: A TECHNICAL CASE REPORT
}

\author{
Marlina Tanty Ramli Hamid ${ }^{*}$, Khairul Azmi Abdul Kadir² \\ ${ }^{1}$ Department of Radiology, Faculty of Medicine University Teknologi MARA (UiTM), \\ ${ }^{2}$ Department of Biomedical Imaging, University Malaya Research Imaging Centre (UMRIC), University Malaya Medical Centre.
}

\section{*Corresponding author:}

Dr Marlina Tanty Ramli Hamid (MRad (UM), MBChB (Manchester)), Department of Radiology, Faculty of Medicine, University Teknologi MARA (UiTM), Sungai Buloh Campus, 47000, Sungai Buloh, Selangor, Malaysia.

Tel:+60361265338. Fax:+60361265164. E-mail: tanty3011@yahoo.com

DOI: https://doi.org/10.32896/cvns.v2n1.13-17

Published: 24.08 .2020

\begin{abstract}
Intracranial dural arteriovenous fistulae (DAVF) are uncommon and account for approximately $1 \%$ of all strokes. All transverse-sigmoid sinus DAVFs require treatment because of the low rate of spontaneous regression and frequent association with aggressive neurologic symptoms. Endovascular embolization which aims for permanent obliteration of the lesion is now the primary treatment modality for all DAVFs. We present a 56-year-old patient with long standing history of intermittent transient ischaemic attack (TIA)-like symptoms. Magnetic resonance (MR) angiography revealed right transverse-sigmoid sinus DAVF and bilateral vertebral arteries occlusion. The patient's symptoms completely resolved upon successful transarterial embolization of the DAVF.
\end{abstract}

Keywords: Intracranial dural arteriovenous fistula (DAVF), Endovascular embolization, Onyx-18.

\section{INTRODUCTION}

Intracranial dural arteriovenous fistulae (DAVF) are uncommon and account for about $10-15 \%$ of all intracranial arteriovenous malformations and approximately $1 \%$ of all strokes ${ }^{1,2}$.

All transverse-sigmoid sinus DAVFs require treatment because of the low rate of spontaneous regression and the more frequent association with haemorrhagic and non-haemorrhagic aggressive neurologic symptoms. Untreated DAVFs of the anterior cranial fossa or the tentorium have $91 \%$ risk of hemorrhage 3,4 .

Endovascular embolization is now the primary modality of treatment for all DAVFs. Other treatment options available include surgery and radiosurgery. The aim of the treatment is for permanent and complete obliteration of the lesion with resolution of clinical symptoms ${ }^{5}$.

We present a patient with a right transversesigmoid sinus DAVF and bilateral vertebral arteries occlusion that presented with long standing recurrent intermittent TIA-like symptoms which completely resolved upon successful transarterial embolization of the DAVF.

\section{CASE REPORT}

A 56-year-old Indian male was admitted with a few year history of intermittent transient memory loss, upper and lower limb numbness, amaurosis fugax, slurring of speech, giddiness and vertigo. These symptoms had increased in severity about 4 weeks before the admission.
No associated loss of consciousness, weakness or headache noted.

Patient was a non-smoker with no other significant medical history. He had a family history of heart disease. He was on Simvastin $20 \mathrm{mg}$ od and Ticlopidine $250 \mathrm{mg}$ bd.

On admission, his Glasgow Coma Scale (GCS) was 15/15 and his pupils were equal and reactive. He was apyrexial with all the vital signs within normal limit (pulse-90bm, blood pressure (BP)-130/90 and oxygen saturation $\left(\mathrm{SpO}_{2}\right)-99 \%$ on room air). Neurological examination was unremarkable and no bruit was heard over the carotid regions.

Clinically, all the blood investigations were within normal limit. Chest radiograph and echocardiogram were also normal. Carotid Doppler study was not requested.

His had a computed tomography (CT) brain scan done in 2005 which showed a small subcentimeter calcified parasagittal meningioma measuring $0.5 \times 1 \mathrm{~cm}$. No significant mass effect was seen on the adjacent brain parenchyma.

Magnetic resonance imaging (MRI) done a few days after the admission showed prominent dilated vessels in the right cerebral hemisphere. MRA confirmed the presence of right transverse-sigmoid sinus DAVF with possible cortico-venous reflux into the right temporal lobe and a prominent draining vein to the anastomotic vein of Trolard were noted. These findings were in keeping with Grade 3 Tranverse-Sigmoid Sinus DAVF. 
MRI also showed multiple small high signal intensity lesions in FLAIR, T2WI and DWI and low signal in ADC in the left periventricular, both occipital lobes and cerebellum in-keeping with acute infarcts. A coincidental finding of bilateral vertebral arteries occlusion with the posterior circulation being maintained by retrograde flow from both posterior communicating arteries (PCOMs).

Cerebral angiography demonstrated a right transverse-sigmoid sinus DAVF that was fed by petrosquamous branch of the right middle meningeal artery and trans-osseos branch of the right occipital artery. There was evidence of cortical venous reflux into the dilated and tortuous vein of Trolard. Cerebral angiogram also revealed gradual narrowing and occlusion of left distal cervical and right intradural segments of both vertebral arteries which was suggestive of dissection. The posterior circulation was maintained by retrograde filling of the patent PCOMs.

Transarterial cerebral embolisation was performed under general anaesthetic two months after admission. 5F $100 \mathrm{~cm}$ Envoy guiding catheter was placed origin of the right external carotid artery (ECA) followed by selective cannulation of the the petrosquamous segment of the middle meningeal artery via Sonic detachable microcatheter $1.5 \mathrm{~F}$ under road mapping. The tip of the catheter was placed within the nidus of DAVF and partially embolized by $3.5 \mathrm{ml}$ of Onyx-18 $(6 \%$ ethylene vinyl alcohol copolymer (EVOH). A 1:3 mixture of Histoacryl to lipiodol were used for embolization of the residual nidus from the occipital branch.

An immediate control angiogram performed post-procedure showed complete obliteration of the nidus and the feeder vessels. The dural sinuses and both intracranial circulation were patent post-procedure.

Cerebral angiogram and MRI done 8 months post-embolization did not show any residual or recurrence of the DAVF. On MRI, the small multifocal infarcts which were seen in the left periventricular, both occipital lobes and cerebellum have also resolved.

There was gradual and continuous improvement of the patient's clinical status.

\section{DISCUSSION}

DAVFs represent an abnormal connection between the dural arteries or the dural branches of the cerebral arteries which is directly shunted into a dural venous sinus or leptomeningeal veins. Their true incidence is unknown since many remained clinically silent or involutes spontaneously ${ }^{1}$.

Most of these lesions are acquired and the commonest predisposing factor appears to be venous sinus thrombosis. Venous thrombosis promotes venous hypertension which opens up microscopic vascular connections within the dura. The multiple fistulous channels that are seen angiographically are thought to represent pathological enlargement of these normal arteriovenous shunts in response to progressive venous stenosis or occlusion thrombosis ${ }^{2}$.
There are several major pathophysiological mechanisms of DAVF that cause cerebral disturbances including steal phenomena, arteriovenous shunt, venous hypertension due to impaired venous return, cerebral hypoxia or ischaemia, cerebral compression by dilated venous aneurysm, sinus obstruction and intracranial hemorrhage due to venous rupture ${ }^{6}$. Our patient has DAVF and chronic bilateral vertebral arteries occlusion. The posterior circulation is being supplied entirely by patent PCOMs. The presence of DAVF resulted in steal phenomena and arteriovenous shunting. This further reduced the blood flow to the already imperfectly perfused posterior circulation which in turn causes cerebral ischaemia. These explain the fluctuating neurological deficits presented by the patient and the resultant multifocal infarcts in the occipital and cerebellar regions.

Awad et $\mathrm{al}^{7}$ demonstrated that the presence of cortico-venous reflux, leptomeningeal retrograde venous drainage, aneurysmic venous extension and galenic venous aneurysm are the most important risk factors for aggressive symptoms which include progressive neurological deficit, increased intracranial pressure and spontaneous intracranial haemorrhage. The overall haemorrhage risk from a DAVF is $\sim 1.5 \%$ per year ${ }^{3,4}$.

The presence of cortico-venous reflux is the ominous sign which warrant urgent intervention and aggressive treatment. These lesions carry increase risk of intracranial haemorrhage with severe neurological complications and generally have poorer prognosis. Spontaneous regression of these AVFs is relatively rare (approximately 5\% of cases) and usually occurs following hemorrhagic events ${ }^{3,4}$. Diagnostic angiogram of our patient revealed a Grade 3 DAVF with all the aggressive features including with antegrade and retrograde venous drainage into the deep and superficial venous system, evidence of cortical venous reflux and aneurysmal venous distension. This clearly defined the patient into a high risk category which warrants prompt and definitive treatment.

Treatment of DAVFs depends on on the patient's clinical presentation, lesion location, angiographic features and the natural history of the lesion. DAVFs have been treated with a variety of approaches, including surgical resection, venous clipping, transcatheter embolization, radiation therapy or a combination of these treatments ${ }^{8}$.

Endovascular techniques are now frequently used as the first therapeutic option for most DAVFs. Transcatheter embolization can be done via an arterial or venous catheter intervention. If a decision is made to treat DAVFs, regardless of the type of procedure, the goal of treatment is total obliteration of the fistula and closing the venous collector is necessary to achieve an anatomic cure. Otherwise, recruitment of collateral flow and continued risk of hemorrhage are likely ${ }^{8}$. In our patient, complete occlusion of the DAVF was achieved via transarterial embolization using Onyx and Histoacryl. 
Onyx-18 is a biocompatible polymer containing ethyl-vinyl alcohol dissolved in an organic solvent (dimethyl sulfoxide). It can be used to treat AVM, aneurysm and DAVFs. Several properties of Onyx make it an attractive permanent embolic agent. It is permanent radiopaque liquid that polymerizes on contact with bloodstream. These allow casting ('plug') of complex vascular networks. It can be injected in a controlled process ("Plug and Push" technique) continuously over a long period of time. It is cohesive but not adhesive: it does not to break off in pieces into the venous system and thereby decreasing the risk of microcatheter retention after embolization. Antegrade filling of the fistula and the retrograde reflux of the Onyx established complete vascular occlusion of the DAVF in just one session. Several studies have shown a low rate of recanalization of Onyxembolized aneurysms even at the 5-year follow-up ${ }^{8}$.

\section{CONCLUSION}

In conclusion, in our patient the DAVF causes steal phenomena which increased blood flow through a lowresistance DAVF vascular bed and divert flow away from the already critical region of the posterior circulation. These causes the TIA symptoms and cerebral ischaemia seen in this patient. The successful obliteration of the DAVFs resolved the steal phenomena and the arteriovenous shunting from the posterior circulation, which in-turn resulted in gradual improvement and resolution of patient's clinical symptoms.

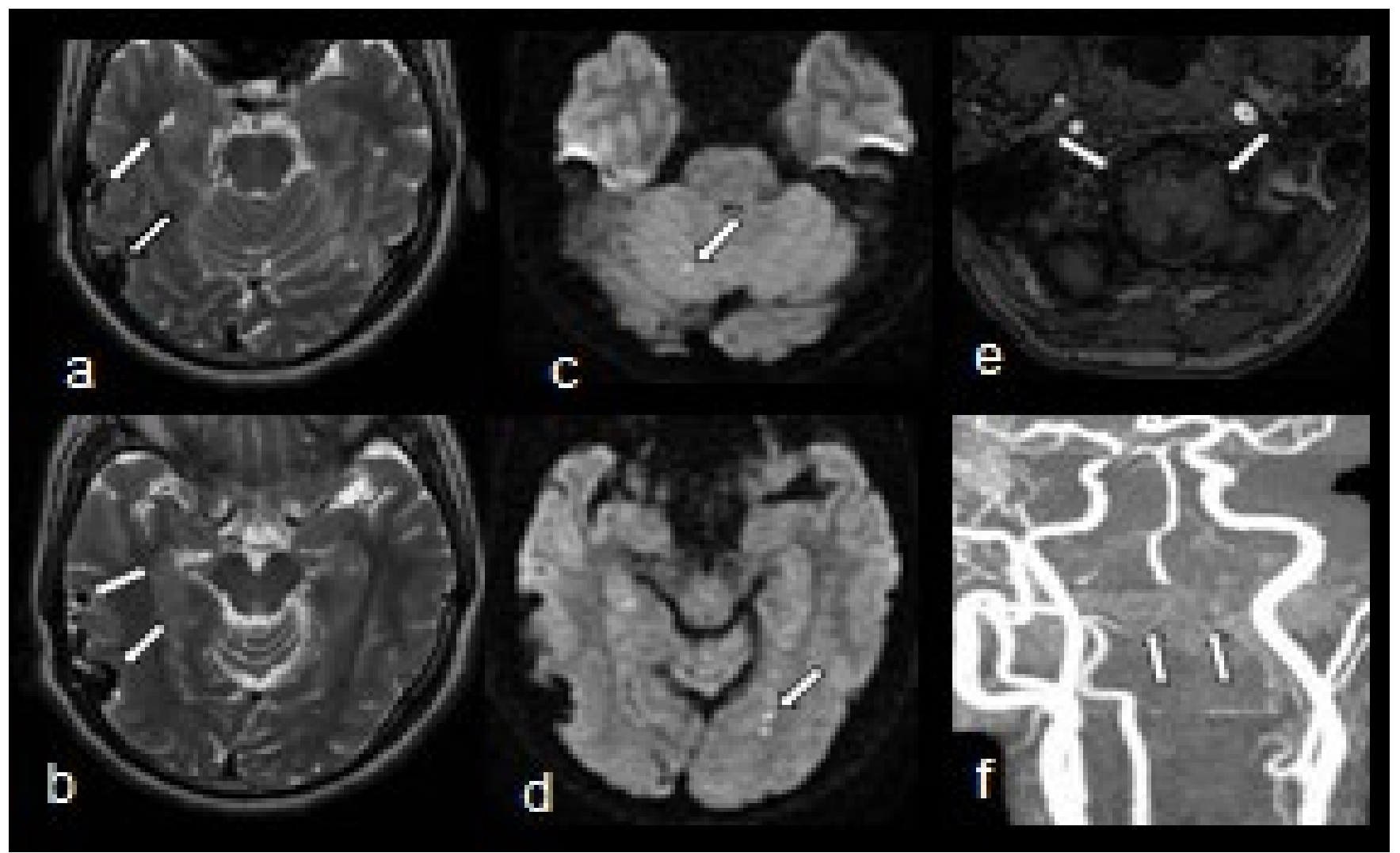

Figure 1- MRI brain images taken 3 days after admission showing:

- $1 a$ and $1 b$ - Axial T2W MRI images showing prominent dilated veins in the right cerebral hemisphere (white arrows).

- $1 c$ and $1 d$ - Axial diffusion weighted MRI images showing multiple hyperintense foci in the occipital lobe and cerebellum (white arrows).

- $1 e$ and $1 f$-Absence of the distal portion of the vertebral arteries bilaterally in-keeping with occlusion (white arrows). 


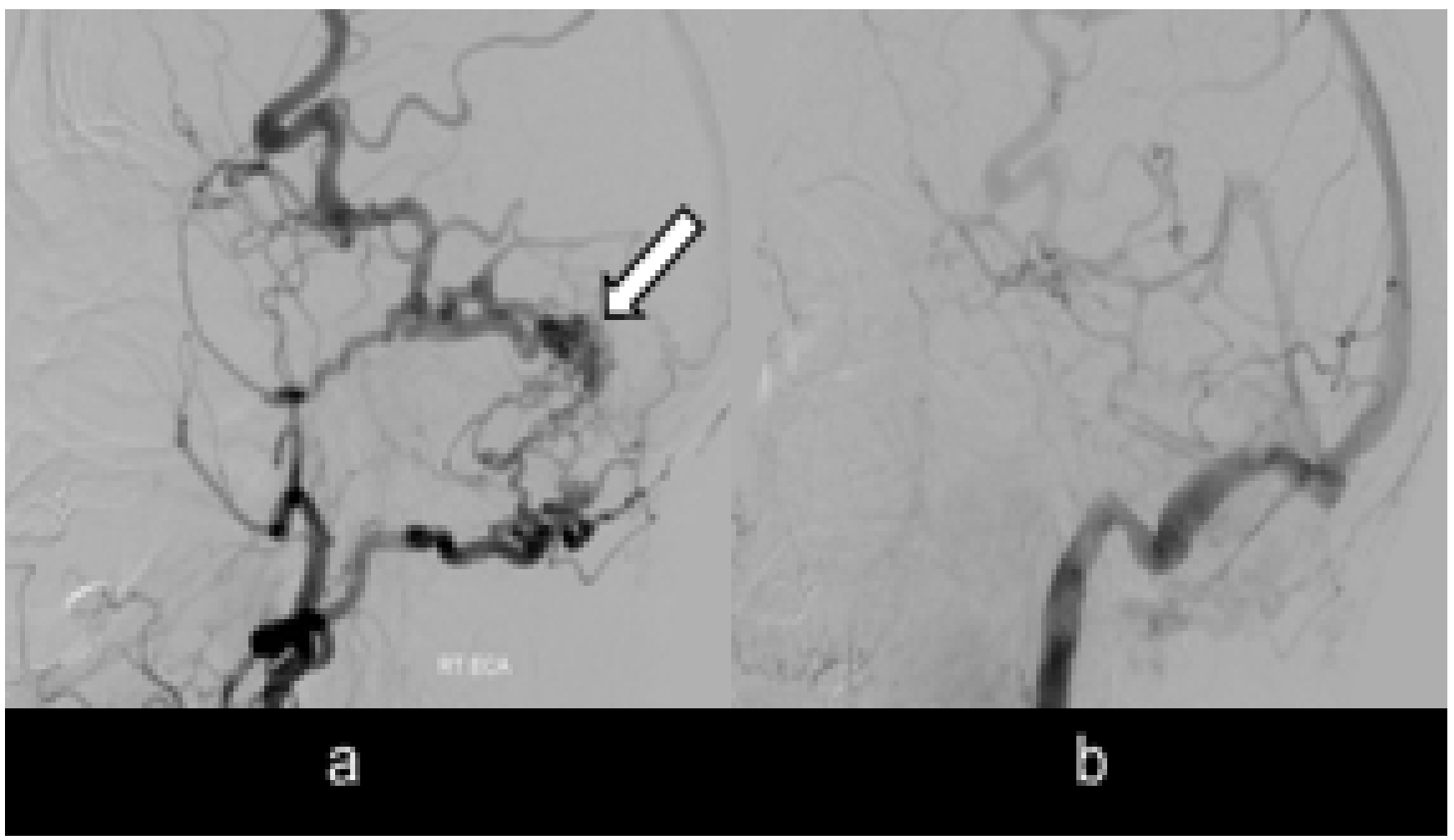

Figure 2:

- a) Lateral projection of the right ECA angiogram reveals a right transverse-sigmoid sinus DAVF (white arrow) fed by the petrosquamous branch of the right middle meningeal artery and trans-osseous branch of the right occipital artery.

- b)There is cortical venous reflux in delayed phase.

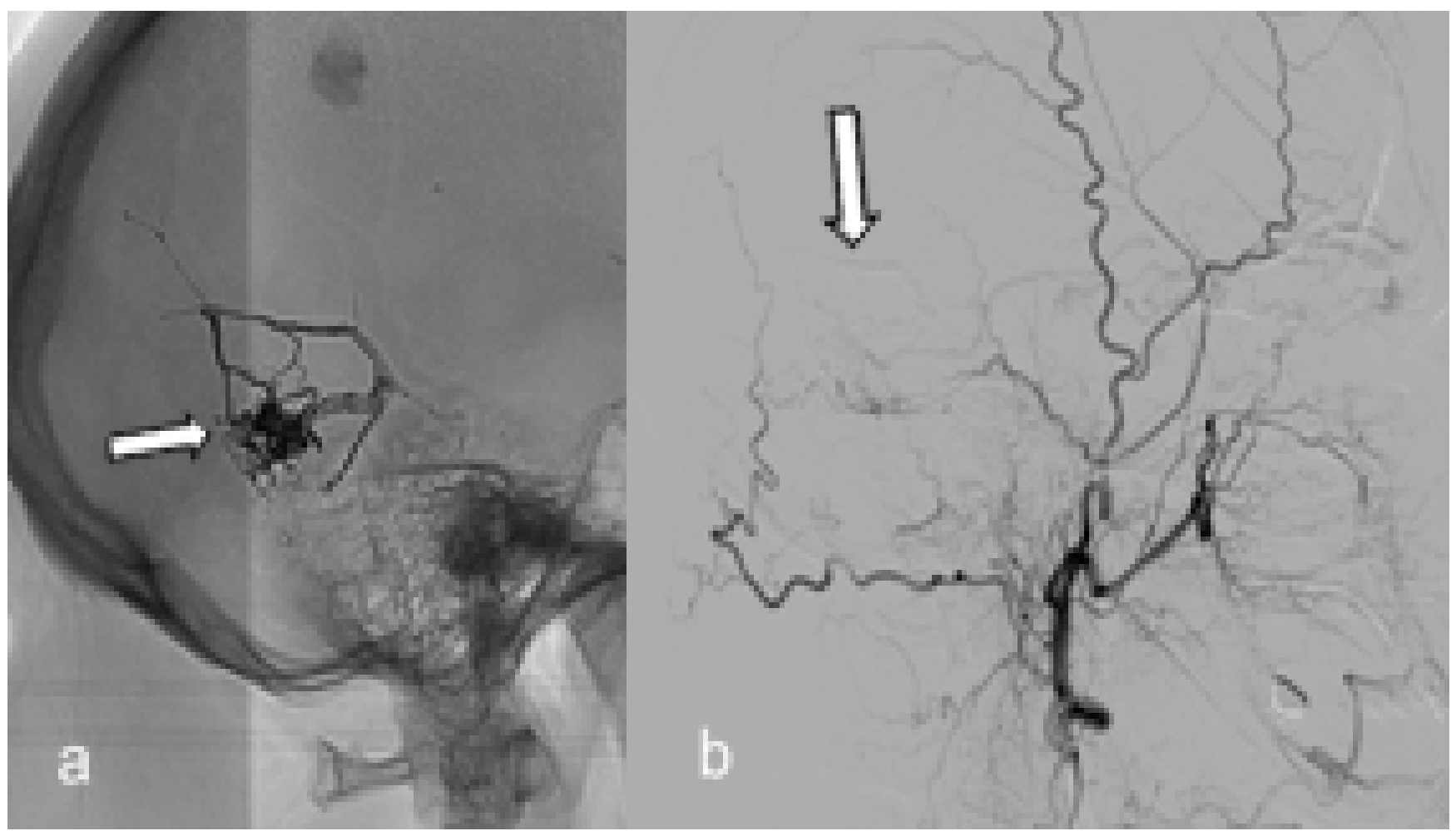

\section{Figure 3:}

- a) Microinjection into the petrosquamous segment of the left meningeal artery filling up the fistula and sinus. Onyx cast filled up the fistula (white arrow).

- b) Post-embolization angiogram image showing complete obliteration of the fistula with Onyx (white arrow) with absence of cortical venous reflux. 


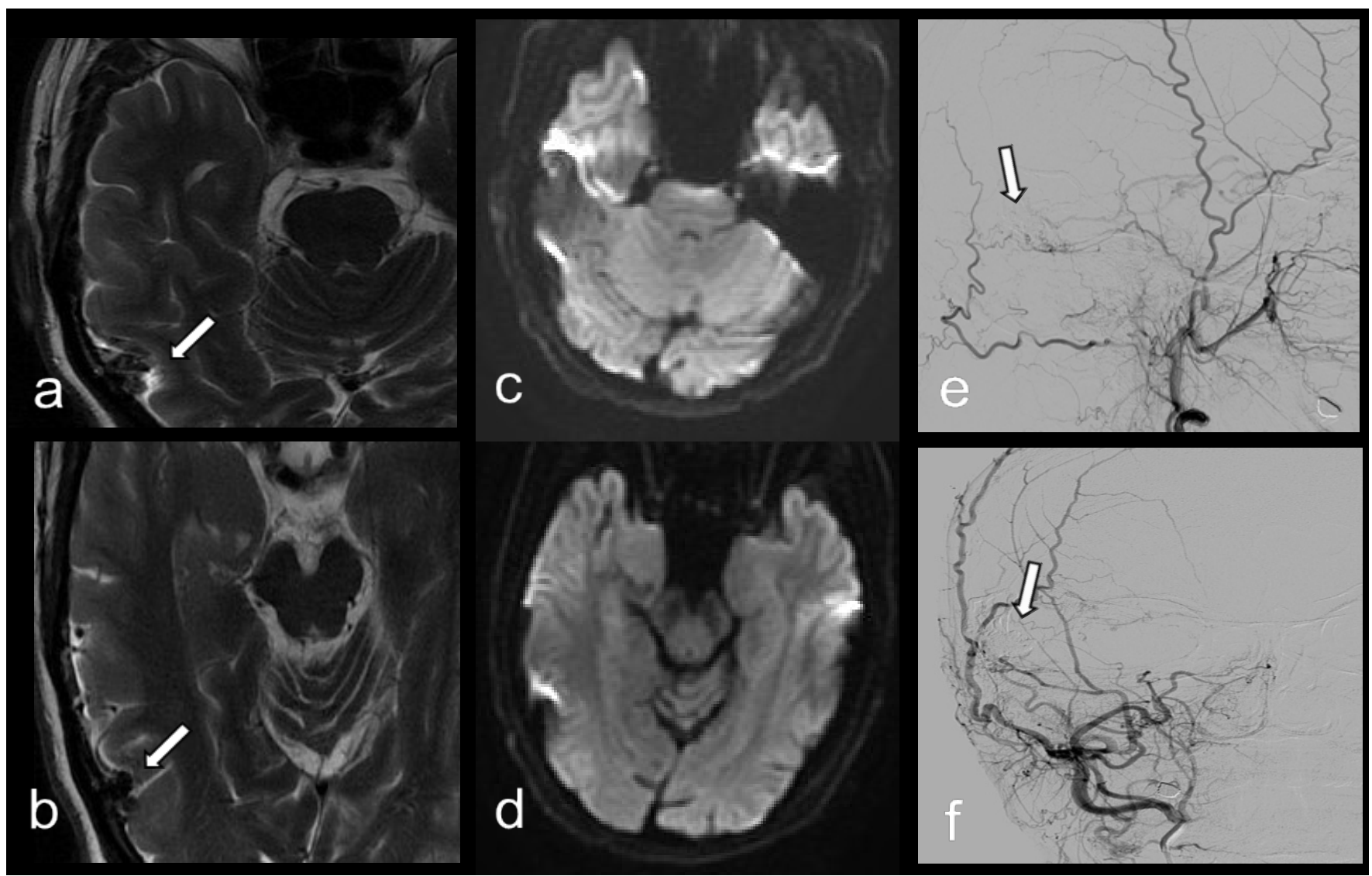

Figure 4:

- $4 a$ and $4 b$ - Follow-up axial T2W MRI images showing no recanalization of the DAVF.

- $4 b$ and $4 c$ - Follow-up axial diffusion weighted MRI images showing resolution of the hyperintense areas previously seen in occipital lobe and cerebellum.

- $4 d$ and $4 e$ - Follow-up cerebral angiogram images showing Onyx cast within the fistula (white arrow) with no evidence of recanalization of the DAVF.

\section{REFERENCES}

1. Adams W, Whitfield P. Intracranial dural arteriovenous fistulas: Management Topic. ACNR 2008:7:3: 10-12.

2. Festa JR, Lazar RM, Marshall RS et al. Dural Arteriovenous Fistula Presents Like an Ischaemic Stroke. Cog Behav Neurol 2004; 17: 1: 50-53.

3. King WA, Martin NA. Intracerebral hemorrhage due to dural arteriovenous malformations and fistulae. Neurosurg Clin N Am 1992; 3: 577-590.

4. Malik GM, Pearce JE, Ausman JI, Mehta B. Dural arteriovenous malformations and intracranial hemorrhage. Neurosurgery 1984; 15: 332-339.

5. Brown RD Jr, Wiebers DO, Nichols DA: Intracranial dural arteriovenous fistulae: angiographic predictors of intracranial hemorrhage and clinical outcome in nonsurgical patients. J Neurosurg 1994; 81: 531-538.
6. Iwama T, Hashimoto N, Takagi Y, et al. Hemodynamic and metabolic disturbances in patients with intracranial dural arteriovenous fistulas: positron emission tomography evaluation before and after treatment. J Neurosurg. 1997; 86: 806-811.

7. Awad IA, Little JR, William PA, Akrawi WP, Ahl J: Intracranial dural arteriovenous malformations: Factors predisposing to an aggressive neurological course. J Neurosurg 1990; 72: 839-850.

8. Saraf R, Shrivsta M, Kumar N et al. Embolization of cranial dural arteriovenous fistulas with ONYX: Indication, technique and outcomes. Indian J Radiol Imaging 2010; 20:1: 26-33. 